\title{
A Comparison of the Local Flap and Skin Graft by Location of Face in Reconstruction after Resection of Facial Skin Cancer
}

\author{
Kyung Suk Lee ${ }^{1}$, \\ Jun Oh Kim ${ }^{1}$, \\ Nam Gyun Kim ${ }^{1}$, \\ Yoon Jung Lee', \\ Young Ji Park', \\ Jun Sik Kim ${ }^{1}$ \\ ${ }^{1}$ Department of Plastic and Reconstructive \\ Surgery, Institute of Health Sciences, \\ Gyeongsang National University Hospital, \\ Gyeongsang National University School of \\ Medicine, Jinju; \\ ${ }^{2}$ Department of Plastic and Reconstructive \\ Surgery, Gyeongsang National University \\ Changwon Hospital, Changwon, Korea
}

\begin{abstract}
Background: Surgery for reconstruction of defects after surgery should be performed selectively and the many points must be considered. The authors conducted this study to compare the local flap and skin graft by facial location in the reconstruction after resection of facial skin cancer.

Methods: The authors performed the study in patients that had received treatment in Department of Plastic Surgery, Gyeongsang National University. The cases were analyzed according to the reconstruction methods for the defects after surgery, sex, age, tumor site, and tumor size. Additionally, the authors compared differences of aesthetic satisfaction (out of 5 points) of patients in the local flap and skin graft by facial location after resection of facial skin cancer by dividing the face into eight areas.

Results: A total of 153 cases were confirmed. The most common facial skin cancer was basal cell carcinoma ( $56.8 \%, 87$ cases), followed by squamous cell carcinoma $(37.2 \%, 57$ cases) and bowen's disease (5.8\%, 9 cases). The most common reconstruction method was local flap 119 cases (77.7\%), followed by skin graft 34 cases (22.3\%). 86 patients answered the questionnaire and mean satisfaction of the local flap and skin graft were 4.3 and $3.5(p=0.04)$, respectively, indicating that satisfaction of local flap was significantly high. Conclusion: When comparing satisfaction of patients according to results, local flap shows excellent effects in functional and cosmetic aspects would be able to provide excellent results rather than using a skin graft with poor touch and tone compared to the surrounding normal skin.
\end{abstract}

Keywords: Skin cancer / Sugical flaps / Skin grafting / Treatment outcome

\section{INTRODUCTION}

The incidence of facial skin cancer has increased gradually with changes in the social and medical environment [1]. In particular, ultraviolet radiation (UVR), an environmental factor, is estimated to be one of the most important risk factors [2]. In most of the reports, the general predilection site of a skin facial cancer is the head and neck because there are many opportunities for these re-

Correspondence: Jun Sik Kim

Department of Plastic and Reconstructive Surgery, Institute of Health Science, Gyeongsang National University Hospital, Gyeongsang National University College of Medicine, 79 Gangnam-ro, Jinju 52727, Korea

E-mail:kjuns@hanmail.net

Received May 20, 2017 / Revised Oct 18, 2017 / Accepted Dec 5, 2017 gions to be exposed to UVR [3]. Current treatment modalities for facial skin cancers and premalignant lesions include surgical excision, electrocautery, curettage, cryotherapy, and irradiation, but surgical excision has been reported to be the best treatment modality for cancer treatment and prevention of recurrence. There are four methods of reconstruction: secondary intention, primary closure, skin grafting, and local and free flaps. Surgery for reconstruction of defects after surgery should be performed selectively and the following points must be considered: surgical method, location of the defect, size, type of the tumor, possibility of recurrence, patient age, health condition, fuctional aspects, and cosmetic aspects [4]. Based on the data from patients diagnosed with 
facial skin cancer at Gyeongsang National University Hospital who underwent complete resection and reconstruction through researches. This study was conducted to compare the local flap and skin graft by location of the face in reconstruction after resection of facial skin cancer.

\section{METHODS}

We retrospectively examined the charts that covered 153 patients who visited the Plastic Surgery Center of Gyeongsang National University Hospital and underwent complete resection and reconstruction after excision of facial skin cancer from January 2010 to December 2014. Clinical information such as the patients' age, sex and location, size according to the reconstruction method were researched. Surgery was performed under general anesthesia or local anesthesia, and inspection of the frozen tissue section was performed in the operating room after complete resection of the tumor. We set the resection margin at $0.5-1 \mathrm{~cm}$ for basal cell carcinoma, Bowen's disease, $1-2 \mathrm{~cm}$ for squamous cell carcinoma, We confirmed that the tumor cells were not found at the edge and the bottom of the facial skin cancer that was resected on inspection of the frozen tissue section; thereafter, we performed reconstruction that suited the surgical location and the resection range.

Also, to compare patients' satisfaction when the local flap and skin graft were conducted by facial location after resection of facial skin cancer by dividing the face into eight areas, the authors conducted a telephone survey on satisfaction with a total of $86 \mathrm{pa}-$ tients with follow-up periods ranging from 12 to 24 months. With 5 points as the full score, the satisfaction (5, very satisfied; 4 , satisfied; 3, modified; 2 , dissatisfied; 1 , very dissatisfied) were investigated to identify results, and if patients were dissatisfied, reasons for dissatisfaction were also examined. Among eight areas of the face, lips, forehead and chin of which only the local flap was conducted were excluded from the satisfaction survey.

Based on the above findings, satisfaction of the local flap and skin graft for each facial area and those for the whole area of face were statistically analyzed through $t$-test. A $p$-value less than 0.05 was considered statistically significant.

\section{RESULTS}

There were 42 men and 111 women, age of the patients ranged from 37 years to 95 years, and the average age was 70 years (Table 1).

Basal cell carcinoma was the most common type of facial skin cancer, which was found in 87 cases (56.8\%), squamous cell carcinoma was found in 57 cases (37.2\%), bowen's disease was found in 9 cases (5.8\%) (Table 2). Nose was the most common location of occurrence and this location was observed in 53 cases (34.6\%), following cheek 49 cases (32\%) and temporal region was the 19 cases (12.4\%), lower eyelid was the 12 cases $(7.8 \%)$, periauricle was the 8 cases (5.2\%), lip and forehead was the sixth 5 cases (3.2\%), chin was 2 cases $(1.3 \%)$.

With respect to the reconstruction method according to the location of the defect that occurred after surgical removal of facial skin cancer, 119 cases (77.7\%) received a local flap, and 34 cases (22.3\%) received a skin graft (Table 3 ).

With respect to the flap method used in reconstruction, Limberg flap and V-Y advancement flap were used a lot (Fig. 1).

Limberg flap was widely used primarily in the cheek, side wall of the nose, forehead, and temporal region, and the V-Y advancement flap was widely used in the nasal alar, nasolabial fold, and lip.

A skin graft was widely used in the nasal tip, and preauricular area, and it was widely used at a location in which the size of the tumor was large (Fig. 2). Additionally, the skin in full thickness skin graft was harvested in the posterior auricular area (19 cases), and supraclavicular area ( 15 cases). The follow up period was 12

Table 1. Patient demographics and diameter data

\begin{tabular}{lccc} 
Characteristic & Local flap & Skin graft & $p$-value \\
Sex (male/female) & $31 / 91$ & $11 / 20$ & 0.26 \\
Age (yr) & 70.62 & 67.61 & 0.18 \\
Diameter (cm) & 1.93 & 2.16 & 0.70 \\
\hline
\end{tabular}

Table 2. The type of facial skin cancer

\begin{tabular}{lc} 
Type & Cases (\%) \\
\hline Basal cell carcinoma & $87(56.8)$ \\
\hline Squamous cell carcinoma & $57(37.2)$ \\
\hline Bowen's disease & $9(5.8)$ \\
\hline Total & $153(100)$ \\
\hline
\end{tabular}


Table 3. Reconstruction methods according to the location of skin tumors

\begin{tabular}{lcccccccccc} 
Method & Cheek & Nose & Temple & Lower eyelid & Lip & Periauricle & Forehead & Chin & Total \\
\hline Flap & 40 & 41 & 13 & 10 & 5 & 3 & 5 & 2 & 119 \\
FTSG & 9 & 12 & 6 & 2 & 0 & 5 & 0 & 0 & 34 \\
\hline Total & 49 & 53 & 19 & 12 & 5 & 8 & 5 & 2 & 153 \\
\hline
\end{tabular}

FTSG, full thickness skin graft.

Table 4. Comparison of satisfaction between local flap and skin graft by facial location

\begin{tabular}{lccc} 
Location & Local flap & Skin graft & $p$-value \\
\hline Cheek & 4.2 & 3.2 & 0.04 \\
Nose & 4.5 & 3.5 & 0.01 \\
\hline Temple & 4.3 & 3.7 & 0.04 \\
Lower eyelid & 4.6 & 3.3 & 0.01 \\
\hline Periauricle & 4.3 & 4.0 & 0.6 \\
\hline
\end{tabular}

Table. 5. Comparison of satisfaction between local flap and skin graft in the whole face

\begin{tabular}{lccc} 
Patient satisfaction & Local flap & Skin graft & $p$-value \\
Satisfaction score & 4.3 & 3.5 & 0.04 \\
\hline
\end{tabular}

month to 24 months after surgery. In all of the flaps and skin grafts, entire necrosis and loss did not occur, and in 5 cases of congestion, 7 cases of local necrosis of the flap, and 3 cases of local skin graft loss was observed.

When analyzing mean satisfaction for each area, scores of the local flap and skin graft were as follow: cheek (4.2, 3.2, $p=0.04)$, nose $(4.5,3.5, p=0.01)$, temple $(4.3,3.7, p=0.04)$, lower eyelid (4.6, $3.3, p=0.01)$, periauricle $(4.3,4.0, p=0.6)$ (Table 4$)$; and mean satisfaction of the local flap and skin graft for the whole face were 4.3 and $3.5(p=0.04)$, respectively (Table 5).

\section{DISCUSSION}

The incidence of facial skin cancer has increased rapidly worldwide over the last decade due to extended life spans and social and medical changes [5]. The rising incidence rate is most likely due to a combination of increased UVR, increased outdoor activities, changes in clothing style, and ozone depletion [6]. In Korea, the incidence of facial skin cancer was reported to be approximately $4 \%$ of all facial skin cancer and this incidence has been increasing steadily [7]. Facial skin cancer have been classified as non-melanoma skin cancer and malignant melanoma. The most common facial skin cancer is basal cell carcinoma and squamous cell carcinoma. In this study, the majority of facial skin cancers were basal cell carcinoma (56.8\%) and squamous cell carcinoma (37.2\%). The incidence of facial skin cancer has been shown to be higher in males than in females due to their more extensive exposure to sunlight [8]. In this study, the ratio of men to women was 0.37:1 and women were predominant, because of increase in average life of women, heightened concern about facial beauty, and the increase in outdoor activity in sunlight are the reasons for this occurrence.

Complete surgical resection is most frequently used in the treatment of facial skin cancers, and the methods used for reconstruction where the location of the defect occurs at this point of time are primary closure, local flap, and skin graft. Factors that should be considered while selecting the reconstruction method are surgical method, location of the defect, size, tumor type, possibility of recurrence, patient age, health status, functional aspect, and cosmetic aspect.

If the defect is not large, primary closure is the most simple and better method, and it has the advantages of a smaller scar and faster healing, but if the defect is large, there may be a dog ear deformity and the surrounding structure can be deformed. A skin graft is useful for facial reconstruction, it is especially useful for the nasal tip, lower eyelid, it can also be used in a wide range of locations of a defect in the temporal region, forehead, and cheek. A cosmetically good result can be obtained by preventing the creation of a defect; furthermore, there is an advantage as it can easily detect tumor recurrence. On the other hand, it is difficult to harmonize with the color and the texture, and there is a disadvantage during curing, as it leaves scars of the plate-like form [9].

The face has a highly developed pilosebaceous unit and a neu- 

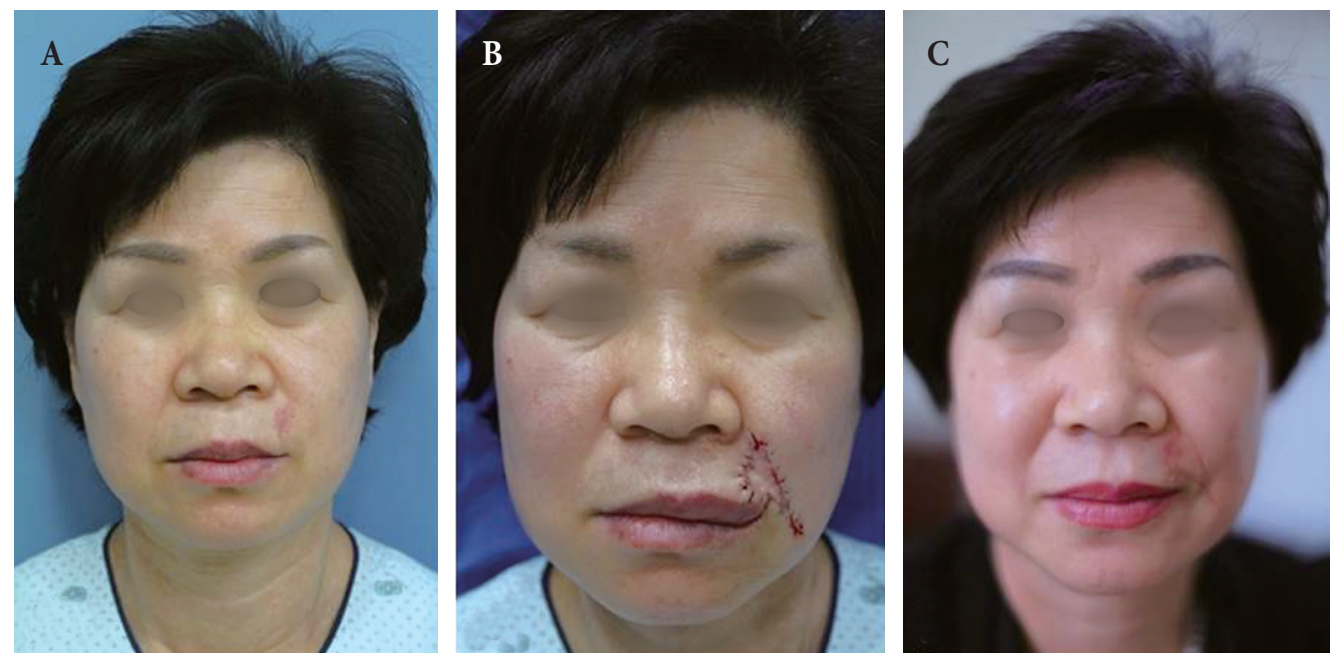

Fig. 1. V-Y advancement flap for reconstruction of the lateral upper lip defect after resection of basal cell carcinoma. (A) Preoperatively, (B) at 3 days postoperatively, $(\mathrm{C})$ at 1 year postoperatively.
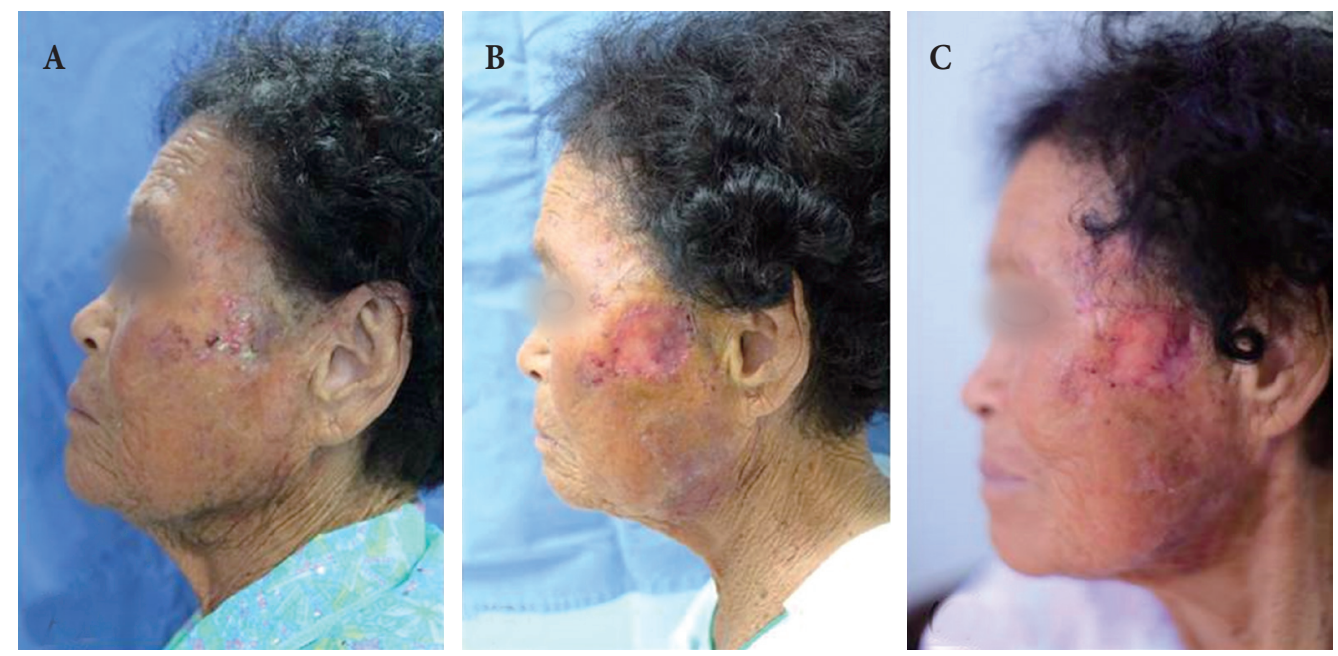

Fig. 2. Full thickness skin graft for reconstruction of the cheek after resection of squamous cell carcinoma. (A) Preoperatively, (B) at 5 days postoperatively, (C) at 6 months postoperatively.

rovascular system, and thus, it recovers fast after surgery, and has the advantage of less occurrence of a scar. Therefore, it is easy to use a flap during reconstruction at the location of the defect after surgical resection of skin cancer. The main advantage of a flap is that it can provide a cosmetic effect by using the donor site having a similar color, texture, and thickness, and a flap has a higher survival rate as compared to a skin graft [10]. In this study also, a flap accounted for the highest frequency among the methods used at the time which was being used at the time of reconstruction at the location of a defect in the face. However, a flap requires an additional incision and movement of tissue, and if the skin incision line is not well hidden under the skin tension line, there will be a disadvantage that an inappropriate scar may persist [11].

Various flaps can be used, and location, size of the defect, and state of the surrounding skin tissue must be considered while selecting the flap method [11]. Limberg flap is the most elegant and simple form, and it can be considered when it is possible to relax the tissue of the surrounding location of the defect, and it is most 
commonly used in the cheek. This not only can disperse tension on the suture as well as on the other potential flaps, but it has an advantage of being able to design the flaps in at least four directions. It allows optimal skin suture matching with the line of wrinkles on the skin, and therefore, it has the advantage of providing an excellent cosmetic result, and it can be mainly used for sidewalls of the nose, forehead, and cheek. However, some incision lines are created vertically on the relaxed skin tension line, and there is a disadvantage that severe tension can act on the location of the secondary defect; therefore, it is important to choose the right flap considering the needs of the operating surgeon [12]. In this study, the side walls of the cheek and nose, the location of the defect on the forehead and the temporal region were found to be mainly reconstructed by using Limberg flap. The other flap method that is commonly used in the cheek is the rotation flap method, and especially, it is often used for reconstruction of a defect located on the inside of the cheek, because the operating surgeon can pull a sufficient margin of skin from the outer side of the cheek. Results after reconstruction with the rotation flap are known to be excellent, but there is a disadvantage that a surgeon needs to design a relatively long incision line to move the flap. Especially when it is applied in young people who can not afford a facial skin scar, a clear scar can be left on their face due to excessive tension, and therefore, appropriate selection in accordance with the needs of the operating surgeon is important [13].

The V-Y advancement flap is particularly useful when a structure or region requires lengthening or release from a contracted state. Less scar tissue, such as trap-door deformity, is formed when this flap is used than when the conventional transposition flap is used in cases involving the alar crease, nasolabial fold, or malar crescent [14]. In this study, it was confirmed that the location of the defect on the lip was reconstructed using a flap.

In this study, there was no case in which the maximum diameter of skin tumor was more than $40 \mathrm{~mm}$, and tumor size of 11-20 $\mathrm{mm}$ showed the most frequent distribution. It is considered important to change people's attitude towards health, increase their interest in the appearance, and pay a visit to the hospital for frequent periodic inspection when the tumor size is small. When selecting the reconstruction method, not only the location of occur- rence but also the tumor size is considered important.

In this study, the authors investigated satisfaction of 86 patients with 5-point scale and found that mean satisfaction for the local flap and skin graft were 4.3 and 3.5, respectively. When investigating satisfaction in each area, the mean score of the local flap was higher than that of the skin graft.

Patients who replied that they were dissatisfied complained of a defect at the donor site and prominence of the scar. Patients who underwent a skin graft complained of the difference in the texture from the surrounding tissue, and the other patients who underwent local flap complained of dissatisfaction with the length of the scar.

We were able to confirm a high level of satisfaction among patients who received Limberg flap which was used on the side wall of the nose and among the other patients who received V-Y advancement flap which was used on the nasolabial fold and the lower eyelid. Satisfaction with the skin graft compared to the flap was slightly reduced, which is considered to be due to some problems associated with the location of the defect on the tip of the nose and the difference in the size of the location of the defect.

Based on the results of this study, we believe that the flap is a method that can be preferentially considered in reconstruction at the location of the defect after excision of facial skin tumor lesion when the tissue around the location of the defect can be sufficiently relaxed. Especially, in this study, effective reconstruction was confirmed in the cosmetic aspect and functional aspect after resection of a lesion less than $30 \mathrm{~mm}$ using Limberg flap and V-Y advancement flap.

Limits of this study were that number of cases of the local flap was larger than that of skin graft, and that the study was conducted with subjective satisfaction of patients only. It is thought that, therefore, photographic assessment should be conducted through operating surgeons or specialists, and then the result of the assessment should be compared with the satisfaction in future studies.

The rate of occurrence of a facial tumor is increasing in response to social and medical changes. Recently, patients have shown a tendency to focus on the cosmetic and functional aspects after surgery for skin tumor removal, the choice of surgical techniques for curing while minimizing postoperative complications 
such as a scar. Based on the results of this study, reconstruction at the location of the defect after facial tumor resection is useful for monitoring tumor recurrence, but rather than using a skin graft that has poor levels of touch and tone compared to the surrounding normal skin, we believe that use of a local flap that provides an excellent effect in and cosmetic satisfaction.

\section{CONFLICT OF INTEREST}

No potential conflict of interest relevant to this article was reported.

\section{PATIENT CONSENT}

The patient provided written informed consent for the publication and the use of their images.

\section{REFERENCES}

1. Glanz K, Schoenfeld ER, Steffen A. A randomized trial of tailored skin cancer prevention messages for adults: project SCAPE. Am J Public Health 2010;100:735-41.

2. Jung YH, Kim SS. A clinical study in malignant skin tumors. J Korean Soc Plast Reconstr Surg 1982;9:377-88.

3. Hwang JI, Kim HS, Park H, Kim JW, Yu DS. A statistical survey of major cutaneous malignant tumors for the last 10 years (2000 2010, North-east Gyeonggido Province). Korean J Dermatol 2011;49:97-105.

4. Mohs FE. Chemosurgery: microscopically controlled surgery for skin cancer. Springfield: Charles C. Thomas; 1978.

5. Hong H, Ji JH, Choi EH. A clinical observation of cutaneous malignant tumors and premalignant lesions in gangwon province over 10 years (1999 2008). Korean J Dermatol 2012;50:95-100.

6. Armstrong BK, Kricker A. Skin cancer. Dermatol Clin 1995;13:58394.

7. Shin JH, Cho S, Whang KK, Hahm JH. An epidemiologic analysis of cutaneous malignant tumors over 15 years (1984-1998). Korean J Dermatol 1999;37:1743-51.

8. Vana J. Epidemiology. In: Helm F, editor. Cancer dermatology. Philadelphia: Lea \& Febiger; 1979. p. 3-15.

9. Summers BK, Siegle RJ. Facial cutaneous reconstructive surgery: general aesthetic principles. J Am Acad Dermatol 1993;29:669-81.

10. Park HJ, Song SK, Whang KK; The Korean Society for Aesthetic and Dematologic Surgery. Aesthetic and dermatologic surgery. Seoul: Hanmi Medical Publishing Co.; 2007.

11. Suh CD, Kim SK, Kim SS. Local flaps in facial reconstruction. J Korean Soc Plast Reconstr Surg 1987;14:417-29.

12. Chu EA, Byrne PJ. Local flaps I: bilobed, rhombic, and cervicofacial. Facial Plast Surg Clin North Am 2009;17:349-60.

13. Cook JL, Goldman GD. Random pattern cutaneous flaps. In: Robinson JK, Hanke CW, Siegel DM, Fratila A, editors. Surgery of the skin. 2nd ed. London: Mosby elsevier; 2010. p. 251-87.

14. Kalus R, Zamora S. Aesthetic considerations in facial reconstructive surgery: the V-Y flap revisited. Aesthetic Plast Surg 1996;20:83-6. 